\title{
Final Scientific/Technical Report Development of Large-Area Photo-Detectors
}

Henry Frisch, PI

Enrico Fermi Institute, University of Chicago

\section{Award Identification}

Agency: DOE

Title of Project: The Development of Large-area, Picosecond Resolution, Timeof-Flight Detectors

Agency Award ID: DE-FG02-06ER41426

University of Chicago TRACS Grants ID Number: 32959

University of Chicago Financial Accounting System Number: 5-29009

Award Period: 1 September 2006 -31 August 2009

\section{Executive Summary}

This proposal requested ADR funds for two years to make measurements and detector proto-types in the context of planning a program in conjunction with Argonne National Laboratory to develop very large-area planar photodetectors. The proposed detectors have integrated transmission-line readout and sampling electronics able to achieve timing and position resolutions in the range of 1-50 psec and 1-10 $\mathrm{mm}$, respectively. The capability for very precise time measurements is inherent in the design, and provides a 'third' coordinate, orthogonal to the two in the plane, for the point of origin of photons or charged particles, allowing 'tomographic' reconstruction in 3-dimensions inside a volume.

\section{Comparison of Actual Accomplishments with Goals and Objectives}

The technical objectives for the effort supported by the ADR grant comprised three areas: a) Psec-resolution capable ASIC design; b) large-area system issues; and c) development of the necessary hardware and software infrastructure to enter the psec time domain. The task list in the proposal comprised:

1. Psec TDC ASIC Prototype and Proof of Principle;

2. TDC Interface Board to DAQ;

3. Psec DAQ System Design;

4. Developing ASIC CAD/Sim Software Infrastructure;

5. Acquiring Psec Testing and Instrumentation Infrastructure. 
In each of these cases we achieved the goals, leading to the present LAPPD Collaboration effort toward large-area psec detectors. In addition, we have created a community working in this area through an extensive series of workshops, talks, and publications.

\section{Project Activities}

The project activities were:

1. System Design. The proposal was to develop a system of up to 10,0002 " $\times$ 2" MCP-based tubes. The DOE_ADR funding allowed us to evolve this to a modular 'Tile-Tray' system that would require a factor of 16 fewer tubes, and a much smaller number of electronics channels.

2. ASIC development, cumulating in the PSEC-4 CMOS design. We successfully designed and tested a 1-channel chip in the proposed IBM $8 \mathrm{HP}$ process, proving that a small university group with no prior ASIC experience can enter the ASIC world.

3. Acquisition of sub-nsec instrumentation, including a a $15 \mathrm{GHz}$ scope and a fast pulser.

4. Printed circuit card design, layout, and fabrication

5. Testing

6. Design and Test software and firmware development

\subsection{Original Hypotheses, Approaches Used}

The original hypothesis was that fast readout of 2"-square MCP-PMT's using micro-strip anodes and ASIC-based waveform sampling was a viable way of achieving high performance. The approach used was to build an ASIC and test card, and to build up the expertise and instrumentation to test electronics at this speed.

\subsection{Problems Encountered, New Approaches}

We did not have the instrumentation to work in the $\mathrm{GHz}$ region; typical $\mathrm{HEP}$ applications work in nanoseconds, but not in picoseconds. We had to build up the necessary instrumentation.

While we successfully made and tested an ASIC in the ultra-fast IBM 8HP SiGe process, we learned that the $8 \mathrm{HP}$ process was too specialized and a psec-capable design would consume too much power. This experience led directly to the successful 6-channel PSEC CMOS series, implemented in the IBM 8RF process.

\section{Products Developed and Tech Transfer}

This grant was the seed that supported work at Chicago on proof-of-principle in several of the key areas essential to the longer-term effort: the transmission 
line readout, the interface to the waveform sampling front-end electronics, and the mechanical and electrical design of the detector devices themselves. It was crucial to the recent (2012) round of successful SBIR and STTR proposals, including the large Tech Transfer Opportunity (TTO) with Incom. 